sets the high standards to follow. The book is large but contains a wealth of basic dermatological thought and fundamental information. The chapters range widely over embryology, keratinization, ultrastructure and histochemistry, carbohydrate, nucleic acid, sterol and lipid metabolism, permeability and blister formation. Many of them are contributed by the acknowledged experts in each field whether from the United States, Europe or Australia.

Philippe Sengel (Paris) describes his elogant exporiments which have thrown so much light on the differentiation of cutaneous appendages in the embryo, and Dame Honor Fell summarizes the present studies on keratinization in organ culture of skin being carried out at Strangeways Research Laboratory. Mercer (Canberra), Brody (Stockholm) and Swanbeck (Stockholm) each give excellent accounts of their own work on the ultrastructure of the components of the horny layer in normal skin, and the deviations which characterize psoriasis. Kligman's (Philadelphia) chapter on the biology of the straturn corneum is especially readable, not only because of his own distinctive style of writing but also because he has directed attention to some of the more neglected biological phenomena in the epidermis. Epidermal cells in the stratum corneum become very much more cohesive to one another than in the basal, Malpighian or granular layers, and yet this cohesion is lost at the surface in such a way that the fully keratinized cells are shed in small flakes which are not normally perceived. An understanding of the mechanism by which such a change occurs should lead to valuable knowledge of many scaly dermatoses.

The organizing committee of this symposium were fortunate to succeed in 'capturing' so many outstanding scientists actively working on the biology of the skin. Drs. Montagna and Lobitz and the Academic Press have produced a volume which is a credit to the participants. There is an author index of the references used in all the papers, and a subject index. Typographical errors are very rare indeed. "Bellberg" for "Bettley" is repeated several times. For a book of this quality it is not overpriced.

$$
\text { C. D. CALNAN }
$$

\section{ATOMIC AND MOLECULAR SPECTROSCOPY}

Spectroscopy and Molecular Structure

By Gerald W. King. Pp. xiv +482 . (London: Holt, Rinehart and Winston, 1964.) $86 s$.

XCELLENT advanced monographs on many different 1 aspects of atomic and molecular spectroscopy are available, and articles in, for example, Annual Reviews of Physical Chemistry and Advances in Spectroscopy, enable research workers to keep abreast of present development. There is, however, at a different level, a need for an introductory text-book suitable for senior undergraduate or postgraduate teaching in chemistry or in physics. Dr. King's book fills some of the gaps admirably.

A very short introduction on spectroscopic technique is followed by chapters on wave-mechanies and the hydrogen atom, many olectron atoms, diatomic molecules, the symmetries, rotations and vibrations of polyatomic molecules, and concludes with a discussion of the electronic spectra of polyatomic molecules and appendixes on operators, solection rules, character tables and physical constants. Each chapter has a short bibliography, and a collection of illustrative problems, some of which are very searching. Mathematical techniques are introduced progressively throughout the book, so that the later chapters are, reasonably, much more difficult than the early ones. However, tho elements of group theory and of matrix algebra, required for the treatment of polyatomic molecules, are introduced both clearly and attractively.

There has necessarily been a rather careful selection of material, so as to limit the book to a convenient length.
Microwave spectroscopy is one of the subjects to suffer. Some description of the techniques would have been welcomed by many students, and the measurement of dipole moments and of nuclear quadrupole coupling constants has provided information of such wide interest that it is surprising that these subjects have been omitted. Again, chemists interested in the propertios of complex compounds will find the discussion of the energy states of transition metal atoms and ions inadequate. The treat. ment of dissociation energies is also very brief (it would have been helpful to have included the paper by R. S. Mulliken (J. Chem. Phys., 33, 247; 1960) in the bibliography at the end of Chap. 6). But, it must be stressed, some selection must be made, and by and large this is a book on the energy-levels of atoms and molecules, and very successful it is too.

The book is well produced and easy to read. The least successful features of the production are the six half-tone reproductions of spectra. There are very few misprints or mistakes. For example, the staternent (p. 75): "Spin-orbit interactions increase rapidly with atomic weight, and are especially large in excited states of heavier atoms" is misleading. These interactions certainly increase rapidly with atomic number, but for a given configuration, say ... $n p$, of a particular atom or ion, the spin-orbit coupling decreases rapidly with prineipal quantum number $n$. Again, p. 211: "The $\Lambda$-type splitting seldom exceeds a few hundredths of a wave-number" should be replaced by "The $\Lambda$-type splitting constants seldom exceed a few hundredths of a wave-number". In the review copy, Fig. 6, 7 (p. 206) was inverted, and there is an obvious error in the printing of problem $8 \cdot 2$ (p. 314 ).

Nearly half the book is devoted to the energy-levels and spectroscopy of polyatomic molecules, and it is this which is its real strength. This is not an easy subject; but the author has succeeded in developing an account which is both lucid and stimulating. This will be recommended reading for many mathematically minded chemists and physicists, undergraduates and postgraduates alike.

R. F. BARROW

\section{BASIC STATISTICAL TECHNIQUES \\ Statistics and Experimental Design in Engineering and the Physical Sciences \\ Vol. 1. Pp. xiv +523. 84s. Vol. 2. Pp. ix $+399.87 s$.} By Prof. Norman L. Johnson and Prof. Fred C. Leone. (New York and London: John Wiley and Sons, Inc., 1964.)

"r THIS book is intended to give students and research workers in science and, particularly, engineering a sound understanding of and facility with basic statistical techniques.

"The material in this book should cover two or possibly three semesters of applied statistics at the upper-division undergraduate level."

These two sentences from the preface indicate the authors' intention. Despite the number of books which aim at fulfilling this function, there is still a need for a good one. The book is long, nearly a thousand pages, and is divided into two volumes. The first volume has three chaptors on probability theory describing the properties of the usual distributions, and also some of the distributions which are of practical interest but are often ignored, and chapters on estimation, testing hypotheses, tosts of significance, regression and correlation which are not particularly well done and appear in almost any other book of this nature. There are chapters on order statistics and non-parametric methods, both of them clearly written, a long and detailed chapter on control charts, and a balanced chapter on the utilization of prior information. The second volume has a long section, more than two hundred pages, on the analysis of variance and the design 